\title{
El modelo de participación ciudadana en salud en Puertollano (España): más allá de la voluntad política y del empoderamiento ciudadano
}

The Model of Citizen Participation in Health Services in Puertollano (Spain):

Beyond Political Will and Citizen Empowerment

Le modèle de participation citoyenne en matière de santé à Puertollano

(Espagne): au-delà des décisions politiques et de l'autonomisation citoyenne

Marta Aguilar Gil y José María Bleda García

\section{OpenEdition}

Journals

Edición electrónica

URL: http://journals.openedition.org/rccs/8293

DOI: $10.4000 /$ rccs. 8293

ISSN: 2182-7435

Editor

Centro de Estudos Sociais da Universidade de Coimbra

Edición impresa

Fecha de publicación: 1 diciembre 2018

Paginación: 123-144

ISSN: 0254-1106

Referencia electrónica

Marta Aguilar Gil y José María Bleda García, « El modelo de participación ciudadana en salud en Puertollano (España): más allá de la voluntad política y del empoderamiento ciudadano », Revista Crítica de Ciências Sociais [En línea], 117 | 2018, Puesto en línea el 03 diciembre 2018, consultado el 19 abril 2019. URL : http://journals.openedition.org/rccs/8293 ; DOI : 10.4000/rccs.8293 


\section{El modelo de participación ciudadana en salud en Puertollano (España): más allá de la voluntad política y del empoderamiento ciudadano}

El modelo de participación ciudadana en salud implantado en el área sanitaria de Puertollano (Castilla-La Mancha, España) se desarrolló durante los años 2008-2011. Fue un modelo innovador, prestigioso y legitimado, basado en la participación activa y deliberativa de diferentes actores implicados en el sistema sanitario. En el año 2011, el gobierno conservador surgido de las elecciones regionales paralizó todas las actuaciones relacionadas con el modelo. A consecuencia de ello se ha estimado conveniente hacer una evaluación a posteriori al finalizar el periodo de implantación, con el objetivo de realizar un análisis riguroso, sistemático y científico sobre lo sucedido durante los años 2008-2015, incidiendo en las debilidades y amenazas del modelo en su aplicación. Para el análisis se ha utilizado una metodología cualitativa, mediante entrevistas en profundidad a actores implicados en la implantación del modelo.

Palabras clave: democracia; España; participación ciudadana; políticas públicas; sistemas de salud.

Palavras-chave: democracia; Espanha; participação cidadã; políticas públicas; sistemas de saúde.

\section{Introducción}

En el año 2005, el gobierno regional de la comunidad autónoma de Castilla-La Mancha (España) puso en marcha un proceso de participación ciudadana en salud con el objetivo de articular un nuevo modelo que superase y complementase los órganos de participación existentes en ese momento. Este proceso culminó con la implementación de un innovador modelo participativo, basado principalmente en la deliberación de diferentes actores sociales (ciudadanos, profesionales y políticos), en que la toma de decisiones era llevada a cabo por todos los sectores implicados, dando un gran peso en la misma a los representantes de los ciudadanos. 
El modelo funcionó, más o menos adecuadamente, durante los años 2008 a 2011. Si consideramos que la participación ciudadana tiene como meta construir sociedades más democráticas y como objetivos consolidar la democracia, limitar el poder de la Administración y comprometer a los individuos haciéndolos más responsables y copartícipes, podemos afirmar que tal fue alcanzado en la implantación del modelo. Asimismo, los objetivos que se pretendían conseguir específicamente con este modelo eran superar el tradicional modelo de asesoramiento y consulta, y hacer viable un mayor grado de participación en las decisiones sobre gestión sanitaria en el área de salud, que también se logró. En cuanto a la finalidad de los diferentes mecanismos de participación (Foro Participativo de Salud, Foro Virtual y Consejo de Participación y Administración), se alcanzaron los objetivos planteados inicialmente: a) deliberar, planificar y elaborar propuestas ciudadanas para el presupuesto económico del área sanitaria; b) debatir y priorizar las propuestas de los dos foros.

El modelo diseñado contemplaba diversos mecanismos -asamblearios, representativos, consultivos y ejecutivos-favoreciendo así una deliberación, tanto fuera como dentro de las instituciones, a través de diferentes órganos de participación donde se planteaba a la Administración las necesidades sanitarias y de salud que se percibían o que se consideraban más importantes para los ciudadanos, a la par de los discursos o propuestas de los políticos y de los técnicos.

Posteriormente a su implantación se llevaron a cabo varias evaluaciones intermedias durante dos años; una realizada por una agencia externa -Instituto de Estudios Sociales Avanzados (IESA/CSIC) - y otra efectuada por profesores de la Universidad de Castilla-La Mancha (UCLM), detectándose en ambas evaluaciones aspectos que había que mejorar, esencialmente los relacionados con el empoderamiento ciudadano, la comunicación entre los actores y la falta de una dotación económica específica para el funcionamiento del modelo (Bleda García y Aguilar Gil, 2016: 114). Las propuestas de mejora que se identificaron en estas evaluaciones detallaron indicadores de las realizaciones efectuadas, así como los éxitos y fracasos del modelo. Estas recomendaciones no pudieron ser asumidas por la Administración pública debido a la paralización/neutralización del modelo por parte de la Consejería de Sanidad (Bleda García y Aguilar Gil, 2014: 151), ya que en el año 2009 se inició la redacción del Plan de Salud y Bienestar Social (2011-2020), en el que se pretendía contemplar la participación ciudadana en salud como una línea transversal que afectaría a todas las líneas estratégicas del mismo.

Por otro lado, las elecciones regionales del año 2011 representan la llegada de un gobierno conservador que en muy poco tiempo hace desaparecer la 
experiencia participativa, favoreciendo así una democracia de baja intensidad. A partir de ese momento se entró en un período de deconstrucción del innovador modelo de participación ciudadana en salud. Transcurridos unos cuatro años, el modelo se desactivó por completo sin ningún tipo de contestación por parte del movimiento ciudadano, la sociedad civil, los profesionales sanitarios o los partidos políticos de la oposición. En el año 2015 hubo nuevas elecciones regionales, lo que significó el regreso de un gobierno socialdemócrata que contemplaba en su programa electoral numerosas acciones de participación ciudadana en salud, pero sin ninguna alusión al modelo participativo que estamos analizando. Estos dos acontecimientos políticos, tan importantes en el sistema sanitario de la región, nos hicieron ver la necesidad de investigar por qué había desaparecido un modelo innovador, legítimo y participativo de gran intensidad democrática, reduciendo casi a la nada la participación ciudadana. Es decir, se vio como necesario conocer las causas de la desaparición del modelo, pues tal como dice Luis López Giraldo (2008: 83): "La comunidad académica es uno de los actores más importantes de un sistema sanitario. Es la conciencia crítica y científica que identifica, estudia, analiza y expresa soluciones a los problemas de salud de toda la población".

Esta investigación social aplicada se ha desarrollado una vez que se ha anulado el modelo y se han iniciado nuevas políticas de participación ciudadana en Castilla-La Mancha, dando así por concluido el modelo participativo anterior, y entendiendo la evaluación como un instrumento para la acción y posibilitador de recomendaciones para mejorar la gestión y ejecución de políticas públicas (Ogando y Miranda, 2001: 17).

El objetivo principal de esta investigación fue analizar el diseño, la implementación y el desarrollo del modelo para identificar qué factores pudieron influir en la desaparición del mismo. Los objetivos específicos son, primero, conocer las opiniones de los principales actores y, segundo, identificar las causas que determinaron la desaparición del modelo favoreciendo la disminución del grado de democracia.

Se partió de las siguientes hipótesis:

a) El modelo se implantó y desarrolló sin empoderar adecuadamente a los actores implicados.

b) La Administración progresista no asumió sus responsabilidades políticas y gestoras, facilitando así su erradicación por parte del nuevo gobierno conservador.

El equipo investigador ha estado formado por investigadores sociales españoles, profesores de las universidades de Sevilla y de Castilla-La Mancha, con una amplia experiencia en investigación sobre políticas públicas en salud. El hecho de ser un equipo externo a la Administración regional conlleva 
ventajas tales como transparencia, objetividad, rigor y comparabilidad (Jorba y Anduiza, 2009: 152). El interés del equipo investigador no solo ha sido académico, sino también político, en el sentido de incidir en la responsabilidad y la transparencia de la Administración.

La metodología usada principalmente ha sido la cualitativa, empleando la técnica de la entrevista en profundidad. Se entrevistó a los actores implicados directamente en la aplicación y desaparición del modelo -ciudadanos, profesionales sanitarios y políticos-, con la finalidad de identificar los diferentes discursos cualitativos para determinar los factores de los éxitos conseguidos y del fracaso del modelo participativo, generando así un gran número de ideas.

\section{Fundamentación teórica y conceptual}

Las tendencias teóricas sobre la participación nos hablan de que esta puede ser por invitación a los ciudadanos por parte de la Administración o por irrupción de los ciudadanos en el poder (Ginés Sánchez et al., 2010). Partimos de la base de que el modelo de participación ciudadana en salud que hemos analizado fue impulsado en sus primeros momentos desde la Administración regional, iniciando el proceso y regulándolo, con el objetivo de fomentar la participación y articular nuevos instrumentos que la facilitasen; es decir, empezó invitando a diversos agentes sociales a participar en la construcción de un modelo participativo. Fue una propuesta sistemática fundamentada y teóricamente sustentada en una democracia más allá de la representativa, donde la administración pública fuese más transparente y permeable a los intereses ciudadanos (Pires y Pineda Nebot, 2011: 261 y ss.; Bispo y Gerschman, 2015: 191).

Ahora bien, aunque el protagonismo inicial fue de la Administración, rápidamente los ciudadanos (movimientos sociales, ciudadanía y sociedad civil) y los profesionales sanitarios (organizaciones profesionales y técnicos) se implicaron activamente en la construcción del mismo mediante el debate con la Administración regional (políticos y técnicos); por lo tanto, se estableció una democracia del tipo deliberativa que, siguiendo al profesor Jesús Ibáñez (1997: 62), se dio a tres niveles:

1. Los elementos. Los ciudadanos, a nivel individual o colectivo, se expresaron democráticamente.

2. La estructura. Las relaciones entre los diferentes actores fueron democráticas, simétricas.

3. El sistema. Se optó por la creación de unas nuevas estructuras que mejoraran la esencia democrática.

Nos encontramos así con un proceso de democracia participativa y directa, con un control de la acción por parte de los ciudadanos, los cuales 
adquirieron una posición primordial en la toma de decisiones, inclinándose de esa manera por una democracia de alta intensidad y acercando a los ciudadanos a la política, a las instituciones y al gobierno (Santos, 2005; Requena Mora y Rodríguez Victoriano, 2017: 4 y ss.). Es decir, el modelo participativo de Puertollano se fundamentaba en las teorías de la democracia deliberativa (Ackerman y Fishkin, 2004; Beste, 2013; Bohman, 2009; Cohen, 2001; Habermas, 2005; Hansen y Rosboll, 2012; Held, 2008; Macpherson, 1997; Robles, 2008), las cuales se consideran un avance en el pensamiento de la democracia que pone el acento en la deliberación, el razonamiento público y la justificación de la acción, con la mayor participación ciudadana posible de una manera individual o colectiva.

Estas teorías consideran que la estrategia fundamental de un proyecto institucional es promover un mejoramiento de la participación ciudadana (en este caso en el ámbito sanitario) a través de un proceso de democracia deliberativa, mejorando las expectativas y las motivaciones de la población, creando nuevos instrumentos o mecanismos de participación y realizando reformas institucionales (Bleda García y Aguilar Gil, 2011: 99). Dicho planteamiento figura en la estrategia del modelo que estamos analizando, complementado con los criterios establecidos por diversos autores (Donoso, 2009; Richer, 2005; Segura, 2010), tales como establecer marcos jurídicos y normativos que faciliten la participación deliberativa, crear mecanismos de participación deliberativa, delegar responsabilidad por parte de la Administración, incrementar el peso de los ciudadanos en los diferentes procesos y aumentar su responsabilidad, fomentar la conformación de redes, empoderar a los ciudadanos, facilitar el acceso a la información, y formular mecanismos de control, rendición de cuentas y gestión transparente.

La investigación se ha centrado fundamentalmente en la búsqueda de indicadores cualitativos, sin olvidar otros cuantitativos. Ogando y Miranda consideran que es "en la última etapa de la vida de una política pública, una vez que desaparece, cuando hay que comprobar si realmente las medidas que se adoptaron surtieron los efectos deseados modificando el estado natural de las cosas, que, en principio, es la razón de ser de toda intervención” (Ogando y Miranda, 2001: 16). La finalidad de esta investigación es detectar, mediante instrumentos cualitativos, qué factores de diseño, ejecución y utilidad influyeron en la erradicación del modelo, cómo se llevaron a cabo y por qué sucedieron esos hechos, adoptando una actitud experimental, buscando las relaciones entre causas y efectos de la política pública participativa con el fin de mejorar las políticas futuras, fortaleciendo así los mecanismos y los procesos de participación ciudadana en salud, en la Comunidad de Castilla-La Mancha. 
Antes de establecer los indicadores o preguntas que utilizaríamos decidimos delimitar ciertos conceptos clave, tales como participación ciudadana, gobernabilidad y gobernanza (AA.VV., 2008: 17 y ss.):

- La participación ciudadana hay que entenderla como un proceso de comunicación entre la ciudadanía y la Administración para tener un conocimiento más aproximado de la realidad social y de las necesidades de la población, con el fin de mejorar la política y la gestión de lo público.

- La gobernabilidad respondería a las diferentes modalidades del poder y a la capacidad que tienen las sociedades para mejorar sus instituciones democráticas y políticas.

- La gobernanza se correspondería con la mejora de la eficiencia de las instituciones democráticas y políticas, incidiendo especialmente en la descentralización del poder, el control de lo social y la eficiencia en la implementación de las políticas públicas, lo que supone también una implicación de diferentes actores en la gestión y en el gobierno.

Otros conceptos clave son:

- Empoderamiento. Entendido aquí como la implicación del ciudadano en la evaluación y planificación de los servicios sanitarios para que sean más eficaces y eficientes, así como en las políticas públicas de salud, pudiendo influir en las agendas de decisión (López Giraldo, 2008: 77).

- Transparencia. Transparencia para explicar la gestión realizada, los resultados conseguidos y los recursos empleados, con la finalidad de lograr una mayor confianza tanto en los ciudadanos como en los profesionales.

Los indicadores o preguntas seleccionados han seguido los siguientes requisitos: homogeneidad, relevancia, claridad, confiabilidad y verificabilidad (AA.VV., 2008: 19). Asimismo, otros autores proponen que para evaluar políticas y planes de salud es conveniente evaluar el proceso, monitorizarlo respecto a cuatro aspectos: actividad, cobertura, calidad y satisfacción (Villalbí y Tresserras, 2011: 18).

Fue así como en relación con las teorías sobre democracia y participación ciudadana en salud, con los conceptos clave, con las características de los indicadores mencionadas anteriormente y con los aspectos del proceso, elegimos los siguientes indicadores pensados específicamente para el análisis: tipos de democracia/de participación, rol de los actores, gobernanza, empoderamiento/conocimiento, comunicación/transparencia y economía/ /financiación funcionamiento. 


\section{Metodología}

La investigación ha consistido en un análisis del modelo para identificar las debilidades y amenazas que contribuyeron a su erradicación, utilizando la técnica de las entrevistas individuales en profundidad.

El análisis llevado a cabo en esta investigación social aplicada lo hemos entendido como un "proceso integral de observación, medida, análisis e interpretación, encaminado al conocimiento de una intervención pública -norma, programa, plan o política- que permita alcanzar un juicio valorativo, basado en evidencias, respecto a su diseño, puesta en práctica, resultados e impactos" (AEVAL, 2010: 13), pero sin olvidar su papel instrumental para hacer más transparente y responsable la acción pública, produciendo de esa manera conclusiones útiles para todos los actores.

El proceso mediante el cual definimos los criterios, preguntas e indicadores para el análisis, tal como hemos explicado anteriormente, contempló tanto las teorías de participación democrática deliberativa, como los conceptos clave; esto nos condujo a unos criterios positivos que se reflejaban en la participación de los actores implicados. Una vez establecidos los criterios relevantes, específicos, que podían responder a las preocupaciones, comportamientos y vivencias de los actores (Jorba y Anduiza, 2009: 155 y ss.; Parés i Franzi y Castellà, 2009: 241 y ss.), se establecieron unos indicadores amplios: tipos de participación democrática, gobernanza y gobernabilidad, empoderamiento y conocimiento de los actores, comunicación y transparencia, y financiación funcionamiento del modelo.

La técnica. Las entrevistas perseguían identificar los diversos discursos de los actores sociales implicados en el proceso de participación ciudadana en salud, objeto de esta investigación, siguiendo así las premisas teóricas de la evaluación naturalista o pluralista que se centran en la importancia de los valores y de las opiniones de la pluralidad de actores (AEVAL, 2010: 85), siendo una técnica "capaz de aproximarse a la intimidad de la conducta social de los sujetos" (Sierra Caballero, 1998: 282). Elaboramos unas preguntas específicas cumpliendo los requisitos formales para este tipo de técnica: sencillas, observables, accesibles, válidas y creíbles para los participantes y los distintos agentes del proceso (Jorba y Anduiza, 2009: 157).

El guion. Para llegar al guion definitivo se elaboró un listado de líneas de indagación con unas cuantas preguntas para cada una de ellas. Posteriormente se llevó a cabo una entrevista piloto, que sirvió para preparar la versión definitiva del cuestionario base de las entrevistas. El guion para la entrevista se estructuró en torno a los siguientes aspectos: tipo de democracia, gobernanza, empoderamiento, comunicación y evaluación. 
Entrevistados seleccionados. Se hizo un muestreo selectivo e intencional escogiendo a las personas que podían facilitar una información profunda y detallada sobre el objetivo de la investigación, pues en este tipo de estudios cualitativos lo que interesa es la comprensión de los fenómenos y de los procesos sociales; también se tuvo en cuenta el rol que desempeñaban en el contexto del proceso. Es decir, el criterio fundamental de su inclusión fue su elevada implicación en la toma de decisiones y su gran conocimiento del proceso.

Se seleccionaron a los entrevistados entre los actores más implicados en el proceso de construcción, desarrollo e implantación del nuevo modelo de participación ciudadana en salud, pues se consideró que ellos eran los más capaces y más dispuestos a dar información relevante, eran accesibles y eran personas muy comunicativas, ya que los actores involucrados asumen un papel fundamental, tanto en la formulación e implementación como en la evaluación de las políticas (López Giraldo, 2008: 77). Se dividieron en tres tipos de actores: tres políticos, dos profesionales y tres ciudadanos, cuyos intereses y prácticas eran diferentes.

Los actores políticos elegidos (E1, E2, E8) -máximos responsables sanitarios- fueron los que más se implicaron en la construcción del modelo: los dos consejeros de Sanidad, bajo cuyo mandato se desarrolló el modelo, y un alto responsable -secretario general- del órgano gestor sanitario, el Servicio de Salud de Castilla-La Mancha (SESCAM). Así pues, eran personas que ejercieron altos cargos de nivel directivo y estratégico en las organizaciones políticas sanitarias regionales.

Los actores profesionales (E3, E4) -máximos responsables del área de salud donde se implantó el proyecto- fueron los que impulsaron el modelo en el área sanitaria de Puertollano: el director gerente del Área de Gestión Integrada de Puertollano y un técnico que desde un primer momento coordinó técnicamente el proyecto dinamizándolo en su implantación. El gerente del área era el principal responsable de la gestión del área sanitaria, que a la vez estaba liderando una experiencia única en Castilla-La Macha donde se unificaron la atención primaria y la especializada. Mientras que el técnico coordinador era el profesional que se puso a tiempo completo, dedicado a la organización del proyecto y su posterior extensión y dinamización.

En cuanto a los ciudadanos (E5, E6, E7) -líderes del movimiento asociativo del territorio donde se llevó a cabo el proyecto-, se seleccionó a tres representantes del movimiento asociativo: una representante de los pacientes y dos representantes de los vecinos, una de las cuales fue la coordinadora del Foro de Participación Ciudadana. Todas ellas estuvieron muy vinculadas al Foro Participativo y muy comprometidas con el movimiento ciudadano de Puertollano. 
Realización de las entrevistas. Antes de efectuar las entrevistas se les informó de los motivos e intenciones de la investigación, fijando con ellos un lugar adecuado y la hora de la cita, así como la intención de devolverles los resultados de la investigación. Posteriormente, unos días antes de la entrevista se les remitió un correo electrónico con un documento -que era un artículo de los autores de esta investigación- publicado en una revista brasileña (Bleda García y Aguilar Gil, 2016), donde se describía el proceso participativo investigado y una evaluación del mismo, con el fin de rememorar el proceso de construcción, implantación y desarrollo del modelo.

Las entrevistas se realizaron en los meses de octubre y noviembre del 2017, en los lugares donde los actores desarrollan sus actividades: a los políticos, en la ciudad de Toledo; a los profesionales, en el municipio de Malagón; y a los representantes de los ciudadanos, en el municipio de Puertollano. Las entrevistas se grabaron para su posterior transcripción escrita y análisis del contenido, llevándose también un diario de campo donde se recogía lo más relevante de la entrevista, así como lo hablado con los actores entrevistados antes y después de realizada la entrevista.

\section{Resultados y discusión}

En este apartado se contemplan las respuestas a las principales cuestiones que hemos planteado como más importantes para el análisis del proceso. Comprende así los análisis de los factores explicativos y las evidencias en las que basaremos nuestras conclusiones y recomendaciones (AEVAL, 2010: 103). En el análisis del proceso nos hemos centrado principalmente en tres aspectos: la valoración cualitativa que los distintos actores tienen sobre el funcionamiento del proceso; la identificación de los factores que han influido positiva y negativamente en el desarrollo del modelo, y las propuestas de mejora y recomendaciones (Ogando y Miranda, 2001: 22).

A continuación se exponen las preguntas sobre las que giraron las entrevistas personales y las respuestas de los encuestados, recogiendo así la percepción individual de los actores sociales, pues tal como dice López Giraldo (2008: 78): "Cada actor social construye su propia percepción de los problemas que le rodean”.

\subsection{Pregunta 1 y respuestas}

El nuevo modelo de participación basado en la democracia deliberativa, aunque estaba legitimado por una normativa aprobada por el gobierno regional, ¿fue asumido por la organización sanitaria (SESCAM) y la dirección política (Consejería)?

Para la mayoría de los actores consultados la organización política sí que asumió el modelo; al más alto nivel sí que fue aceptado, entre otros, 
por el propio presidente del Gobierno de la comunidad autónoma (E3), ${ }^{1}$ "se aprobó una normativa legal (Decreto 61/2007), que fue impulsada por la Consejería de Sanidad y por el SESCAM apoyando las iniciativas previas y el desarrollo posterior" (E8); ${ }^{2}$ pero los niveles más inferiores no se comprometieron, no se consiguió su connivencia o complicidad, aunque fuera mínimamente, pese a que se intentó.

Los actores políticos y ciudadanos estiman que en el diseño y la construcción sí que existía una responsabilidad política, la cual empieza a decaer con el siguiente consejero de Sanidad, paralizando de esta manera la extensión del modelo a otros lugares de la comunidad autónoma, probablemente debido a que comienza un periodo de restricción presupuestaria a consecuencia del inicio de la crisis económica global. Otro factor pudo ser el temor a que se incrementaran las demandas ciudadanas, lo que podría suponer un incremento económico en el presupuesto sanitario, que no podría ser contraído: "Temor ante las demandas de la democracia deliberativa, no se van a poder satisfacer las nuevas demandas" (E1), había "miedo en la organización política sobre las peticiones ciudadanas... se les hizo hasta grande, no se esperaban que funcionara tan rápido" (E4). ${ }^{4}$ La elite gestora del SESCAM, formada por más de 50 personas, no debatió el nuevo modelo participativo, aunque tampoco lo cuestionó públicamente: "lo escucha, es muy bonito, está muy bien... pero no levanta ningún interés entre los directivos" (E2).5 El mensaje político en defensa de la democracia deliberativa era atractivo para los propios políticos: "encajaba bien en la filosofía de la Junta y de la Consejería de favorecer la participación ciudadana" (E8); un lema de aquella época era: "El paciente es el centro del sistema". Para fundamentarlo se inició el nuevo modelo participativo, pero la realidad fue que gran parte de los directivos políticos y gestores no creían en el mismo: "Por debajo del consejero no se lo creía nadie" (E4), con la excepción del gerente del Área de Puertollano que "facilitó todo lo necesario para el funcionamiento del Foro" (E5), ${ }^{6}$ "hacía caso a las peticiones ciudadanas" (E6). ${ }^{7}$

Para los ciudadanos el proyecto fue muy atractivo en un principio, confiaron plenamente en las organizaciones política y gestora, pero "cuando no hubo presupuesto económico para el funcionamiento del Foro, pues empecé a no creer en el compromiso" (E5). Los ciudadanos sí que se implicaron en

\footnotetext{
${ }^{1}$ E3: Álvaro Santos Gómez, gerente de Área Sanitaria.

2 E8: Fernando Lamata Cotanga, consejero de Salud y Bienestar Social (2008-2011).

3 E1: Roberto Sabrido Bermúdez, consejero de Sanidad (2004-2008).

${ }^{4}$ E4: César Fernández Carretero, técnico coordinador.

5 E2: Rafael Peñalver Castellano, secretario general del SESCAM.

${ }^{6}$ E5: Trinidad Sánchez Pérez, representante de la asociación de vecinos.

${ }^{7}$ E6: Mariano Rodríguez Rodríguez, representante de la asociación de vecinos.
} 
el proyecto, apostaban por una democracia deliberativa, los políticos no: "A los políticos les molesta que los ciudadanos les demos ideas, les molesta que les cuestionemos... la presencia política distorsiona... yo les dejaría completamente al margen en un proyecto participativo" (E5).

De las opiniones anteriores se deduce una mínima implicación de las organizaciones políticas y sanitarias en el desarrollo del modelo participativo, poniendo más el acento en un proyecto impulsado por unos pocos actores, que son los que ejercen el liderazgo inicial, pero que cuando entran otros actores aquellos ya no se implican de la misma manera y, por consiguiente, el modelo entra en decadencia, propiciando de este modo su desaparición posterior con la entrada de un gobierno conservador en la comunidad de Castilla-La Mancha.

Una posible interpretación de esta, casi única, implicación de los actores políticos y gestores que impulsaron inicialmente el proceso, está relacionada con lo que apunta Segura (2010: 60): "Probablemente el renovado interés en el fomento de la implicación de ciudadanos y pacientes en la escena de la política sanitaria tiene que ver con la necesidad de contención de los costes públicos y con la de compartir las responsabilidades en la utilización de los servicios sanitarios", que pudo traducirse en unos intereses políticos coyunturales y personales, impidiendo así la asunción de un modelo por parte de la organización política y gestora. $\mathrm{O}$, tal vez, como opinan otros autores, muchas organizaciones no pueden hacer frente a todas las posibilidades de participación que se le atribuyen (van de Bovenkamp et al., 2010: 73). A ello habría que añadir la frustración de los ciudadanos ante esa falta de implicación, pues tal como ha sucedido en otras experiencias deliberativas, sus expectativas fueron mayores que los resultados obtenidos, y la participación sin la cooperación de los servicios sanitarios y el apoyo financiero y organizacional lleva consigo una disminución en la participación ciudadana (Serapioni y Duxbury, 2012: 488; Matos y Serapioni, 2017).

\subsection{Pregunta 2 y respuestas}

¿Estaban preparadas las organizaciones sanitarias, sociales y profesionales para asumir un control compartido en la toma de decisiones?

Para los profesionales sanitarios la participación ciudadana no entra en sus prioridades; en aquel momento estaban preocupados por "sus retribuciones, nuevos servicios, carrera profesional, incremento de personal... la participación ciudadana no les interesaba" (E1). En su gran mayoría no reconocen la democracia deliberativa, consideran que no tienen que compartir con ellos la gestión; a lo que habría que añadir "su ideología, que en su mayoría es conservadora, lo que supone estar en contra de los fines de 
la democracia deliberativa... Los profesionales médicos y de enfermería, así como las organizaciones sindicales no apoyan la democracia deliberativa en salud" (E2). No obstante, hay que reconocer que sí que hay profesionales sanitarios progresistas que se implican apoyando los modelos participativos.

Al principio, las asociaciones que formaban parte del Foro Participativo estaban a la expectativa, no acababan de creerse ese nuevo modelo de gestión, de democracia deliberativa. Posteriormente, cuando empezó a desarrollarse, vieron que debían comprometerse en la gestión de su área sanitaria; en ese momento "hubo asociaciones que se mostraron reticentes a asumir una corresponsabilidad en la gestión... ¿Que yo tengo que decidir? No, decide tú (profesionales, gestores, políticos), que para eso cobras" (E1). Aun reconociendo sus temores, los ciudadanos asumieron su responsabilidad en la toma de decisiones y formaron parte de los órganos consultivos y directivos; de hecho, todas las acciones aprobadas en esos órganos fueron remitidas a la dirección del SESCAM, ejecutándose la mayoría de ellas "tal vez porque no suponían un gran cambio en la gestión y no suponía un incremento económico en el presupuesto anual" (E1). La desconfianza inicial fue superada muy pronto, sobre todo por los actores sociales más implicados en el desarrollo del proyecto.

Uno de los grandes problemas en la asunción de responsabilidades por parte de los ciudadanos fue la rivalidad entre ellos: "cada uno tiraba hacia lo suyo" (E7). Se reconocía su espíritu de lucha, ya que el movimiento asociativo de ese territorio es combativo y tiene tras de sí una historia ejemplarizante, pero en un modelo organizativo donde hay que olvidar los intereses sectoriales y pensar en lo común, la experiencia fue, principalmente, conflictiva; surgieron las rivalidades y las envidias. Sin embargo, con el paso del tiempo, "cuando empezaron a ver que iban consiguiendo actuaciones que redundaban en lo común, fueron implicándose más en el proyecto” (E4). Es decir, era un cambio cultural en la participación ciudadana a la que estaban acostumbrados históricamente: "Es un cambio cultural de participación, tenemos que olvidar lo nuestro, debemos pensar en los otros" (E5). A esto habría que añadir que los componentes del Foro no conocían el funcionamiento del sistema sanitario: "Les faltaba información, eran personas comprometidas socialmente, pero no tenían ni información, ni formación" (E6), o tal como expresa un actor del sector ciudadano, "éramos incapaces de asumir responsabilidades" (E7); ${ }^{8}$ cuestión que es muy importante, tanto al principio como durante el desarrollo del proyecto, sobre todo en un sistema tan complejo como es el sanitario.

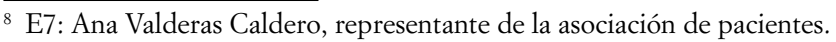


Este nuevo modelo conllevaba también un cambio en la cultura participativa: "Lo que no es fácil y se precisa de más formación y sensibilización, tanto de las organizaciones sociales como de los profesionales. Es un proceso que se había iniciado, pero que quedó truncado" (E8).

De todo lo anterior y teniendo en cuenta investigaciones empíricas internacionales, podemos deducir que aunque la participación brinda a los ciudadanos y a los pacientes muchas oportunidades de implicarse, también causa importantes tensiones (van de Bovenkamp et al., 2010: 73). Al mismo tiempo, la evidencia científica nos dice que existen muchos mitos sobre la necesidad de compartir la toma de decisiones, pero que la realidad es todo lo opuesto; sin embargo, esto no es óbice para lograr ese cambio de paradigma, pues es necesario, sobre todo, para optimizar el uso de los recursos escasos e integrar los enfoques centrados en el paciente (Légaré y Thompson-Leduc, 2014: 284).

\subsection{Pregunta 3 y respuestas}

Antes de implantar el modelo, ¿se debería haber formado convenientemente a los ciudadanos y a los profesionales ante los nuevos compromisos?

En la implantación del modelo se partió de que era necesario empoderar convenientemente a los ciudadanos y a los profesionales; por ello, desde un primer momento se planteó la información del nuevo modelo a los miembros del Foro, así como una formación adecuada a los nuevos escenarios en los que se iba a participar. Sin embargo, la organización gestora, el SESCAM, que era quien iba a asumir y liderar la puesta en marcha del nuevo modelo, no se implicó todo lo que hubiera sido deseable en los aspectos formativos de los ciudadanos y los profesionales: "La organización gestora no se creyó el nuevo modelo, lo veían como un proyecto del consejero de Sanidad y dos más" (E1). En su defensa, quizás habría que tener en cuenta que eran los primeros momentos de asunción de las competencias sanitarias en la región, "por lo que estaban en otras cuestiones que ellos consideraban más prioritarias, fundamentalmente las asistenciales" (E1).

Desde el primer momento se desarrollaron acciones de formación dirigidas a los ciudadanos. Estas acciones fueron muy bien acogidas por los miembros del Foro, sirvieron para tener una percepción más creíble del modelo: "En las asambleas se empezaron a debatir las necesidades y a priorizar las decisiones... se comenzó a pensar en lo común" (E4). Sin embargo, hubo dificultades para que los actores acudieran a esas actividades formativas, fundamentalmente por el costo económico de los desplazamientos, la lejanía del lugar residencial o el horario en que se realizaban: "Es necesario financiar esas horas de formación" (E7). 
En cuanto a los profesionales, la mayoría carecía de información y formación sobre el nuevo modelo; incluso para los que tenían cierto conocimiento, la participación ciudadana en salud no se encontraba entre sus prioridades e incluso la rechazaban: "¿Que los ciudadanos me van a decir lo que tengo que hacer?" (E4). Uno de los gestores opina que "los profesionales se tenían que haber involucrado más en el modelo, incluso en su propio interés" (E4).

Todos los consultados consideran la conveniencia del empoderamiento de todos los actores implicados, para lo que hubiera sido necesario contar con una partida económica dedicada exclusivamente a tal fin, sobre todo para los componentes del consejo de participación y administración. El empoderamiento de los actores es clave; se hicieron actividades con esa finalidad, pero escasas; se debería haber invertido mucho más en formación.

Las teorías clásicas consideran fundamental el empoderamiento de los actores participantes; sin embargo, un paso más allá es el aportado por Robert White (2004: 22) cuando afirma que el empoderamiento valora la identidad propia y la cultura local, debe orientarse hacia el servicio de la sociedad y debe ser considerado como un derecho humano. A ello habría que añadir el aumento continuo de ciudadanos que utilizan internet para su información y formación en salud, lo que supone un reto para los profesionales sanitarios al encontrarse con ciudadanos informados y facultados (Santana, 2011).

\subsection{Pregunta 4 y respuestas}

$¿$ Los diferentes actores afectados (políticos, profesionales y ciudadanos) por la implantación del modelo estaban informados razonablemente sobre el nuevo modelo de participación? ¿Hubo una adecuada comunicación a la sociedad en general sobre el nuevo modelo y las acciones que se llevaron a cabo posteriormente?

Consideran que la información debería haber sido clave, pero la realidad es que el nuevo modelo no se comunicó adecuadamente a los ciudadanos en general, aunque sí a los miembros del Foro Participativo, a través de reuniones, cursos, página web, prensa, radio, seminarios y congresos. Sobre todo se hizo un gran esfuerzo en informar acerca del modelo en todos los municipios que integran el área sanitaria de Puertollano. Sin embargo, estos últimos no se implicaron en su totalidad, no hicieron suyo el modelo; a lo que habría que añadir la escasa implicación de las organizaciones políticas en su papel de difundir y propagar el modelo. "Hubo una información razonable, aunque siempre mejorable. De hecho, muchas asociaciones 
ciudadanas demandaron participar en el proceso. También los ayuntamientos y los medios de comunicación fueron informados" (E8).

Habría que destacar que todas las actuaciones que se llevaban a cabo en los diferentes órganos participativos del Foro, eran comunicadas a todos sus miembros a través de diferentes medios, tales como las actas y la página web -diseñada específicamente para darle visibilidad e informar a toda la ciudadanía-, aunque "la sociedad en general no tenía información de lo que se hacía en el Foro... fuera del Foro no se tenía conocimiento de lo que se hacía" (E5).

En el ámbito político y de gestión sanitaria se deberían haber realizado actuaciones de información, de divulgación del nuevo modelo participativo dirigidas a los responsables políticos, sociales y sanitarios de la región; incluso se "tenía que haber informado del modelo fuera del territorio de Castilla-La Mancha... habría hecho falta una campaña informativa en toda regla" (E2). Los procesos de comunicación en salud son necesarios, ya que pueden ayudar a potenciar y alcanzar los objetivos de las organizaciones sanitarias; hay que facilitar tanto la comunicación interna como la externa, ascendente, descendente y transversal (Bustamante Ospina, 2013). Asimismo, otros estudios a tener en cuenta nos muestran que la mala comunicación entre los diferentes grupos implicados es una barrera y que la actitud y comunicación basada en un respeto mutuo es una ventaja (Atanasova-Pieterse y Kerekovska, 2013).

\subsection{Pregunta 5 y respuestas}

Desde su perspectiva, ¿cuáles pudieron ser las tres principales causas del fracaso del modelo?

Todos los actores consultados coincidieron en que el modelo no ha fracasado, sino que no ha interesado que funcionase y, sobre todo, que no hubo tiempo suficiente para desarrollarlo -insuficiente sedimentación-, ya que mientras estuvo en marcha fue operativo; las evaluaciones que se hicieron así lo manifestaban. Sin embargo, están de acuerdo en que hubo errores que impidieron que su desarrollo fuera el más adecuado e identifican como más importantes los siguientes:

- Consideran que el factor que más influyó en el desarrollo del nuevo modelo fue la falta de implicación de las organizaciones políticas (Consejería de Sanidad) y la gestora sanitaria (SESCAM), que sin embargo fueron las que lo elaboraron, aprobaron una norma legal para su implantación y pusieron los primeros recursos para su desarrollo, lo que supone una discordancia entre el plano legal y la práctica real. 
- La segunda causa más citada es la escasa información y formación de todos los actores implicados en el desarrollo del modelo, así como de la ciudadanía en general.

- La tercera causa es la ausencia de una partida económica finalista para el funcionamiento del Foro de Participación en salud, lo que no es óbice para que los ciudadanos hubieran defendido el proyecto, ya que lo consideraban muy positivo.

Estas causas también han sido identificadas en otras experiencias participativas (Serapioni y Romaní, 2006; Mosquera et al., 2009; Serapioni y Duxbury, 2012).

De las respuestas de los entrevistados podemos deducir que el modelo participativo en salud de Puertollano cumplió, al comienzo de su implantación, con los principios de "buen gobierno", que recoge el Libro Blanco de la Gobernanza Europea (apud AEVAL, 2010: 17), tales como apertura (comunicación activa), participación (amplia participación ciudadana), responsabilidad (de los actores implicados), eficacia (producción de resultados) y coherencia (políticas y acciones coherentes y comprensibles). Sin embargo, durante el poco tiempo en que estuvo desarrollándose las responsabilidades de los actores van decayendo, incumpliendo así las responsabilidades asumidas inicialmente o que les correspondían entre sus deberes y obligaciones.

Tras el análisis de las entrevistas hemos identificado las siguientes debilidades y amenazas del modelo participativo.

1. Debilidades (internas):

a) De comunicación. Escasa comunicación interna y externa, escaso desarrollo del plan de comunicación existente en el Foro Participativo, insuficiente transparencia de las acciones efectuadas, y deficiente comunicación entre los órganos participativos y la organización gestora.

b) De cultura. Diferentes culturas participativas que varían según el grupo concreto de actores y desconocimiento por parte de los ciudadanos de la cultura de la organización gestora y del funcionamiento del sistema sanitario.

c) De comportamiento. Desconfianza entre los actores implicados, liderazgo muy personalizado y focalizado, y poca o nula implicación de la organización gestora y de los profesionales.

d) De formación. Deficiente empoderamiento de todos los actores involucrados en el proyecto.

2. Amenazas (externas):

a) De comunicación. Desconocimiento del modelo participativo por parte de la sociedad. 
b) De cultura. Choque cultural entre la organización gestora y los ciudadanos; resistencia al cambio por parte de los profesionales, los gestores y los políticos, que les lleva a una escasa implicación en el proyecto; y la preocupación prioritaria de los profesionales y los gestores es su actividad asistencial y de resolución de problemas más acuciantes.

c) De comportamiento. Ausencia de liderazgo de los responsables políticos y gestores que continuaron con el proyecto inicial, pues el primer cambio político después de aprobar la norma condujo a una menor implicación en el mismo, tanto de los nuevos políticos como de los nuevos gestores de la organización sanitaria.

d) De economía. Incumplimiento de la inclusión en los presupuestos económicos anuales de las actuaciones aprobadas por los ciudadanos en los órganos creados para la toma de decisiones, lo que supone un control inadecuado de los procesos económico-administrativos derivados de esas decisiones; y la reducción de los presupuestos destinados a la sanidad pública debido a la crisis económica regional y estatal.

\section{Conclusiones}

La importancia de las políticas públicas es fundamental en las sociedades contemporáneas. Un sector importante de las mismas desea aumentar la participación directa en la construcción de dichas políticas; sin embargo, la realidad va por otro camino, agravado aún más por la incertidumbre de los procesos políticos, ya que estos siempre están sujetos a cambios debido a las diversas coyunturas económicas, políticas o sociales, por lo que es difícil ser fiel a los compromisos adquiridos (Manin et al., 2006: 113 y ss.).

En principio, seguimos considerando que es necesario analizar los procesos participativos, pues hay pocos estudios empíricos que puedan ayudar a seguir avanzando en la construcción del paradigma teórico de democracia. El debate entre teoría y praxis, lo normativo y lo positivo, lo políticamente pragmático y lo analítico crítico reflexivo sigue en pie (Pires y Pineda Nebot, 2011: 274). El paradigma teórico de democracia es conflictivo a la hora de aplicarlo a realidades sociales concretas, ya que es difícil desarrollar el discurso académico y/o teórico; ambas cuestiones se encuentran muy distanciadas.

La insuficiente sedimentación del proyecto podría ser una causa básica en el escaso desarrollo y nula exportación del modelo a otras áreas sanitarias. Habría que resaltar el corto periodo de tiempo que estuvo desarrollándose el modelo de Puertollano; estaban sentadas las bases, pero no dio tiempo 
a ejecutar todo lo programado ni a corregir los defectos identificados en las evaluaciones anteriores. Un cambio cultural, tal como era la meta del proyecto, necesita más tiempo; se sembró, pero no dio tiempo a que creciera un mínimo. Por lo tanto, más que un problema de diseño institucional, hubo factores que pudieron influir en el desarrollo del modelo participativo, tales como la cultura de la organización y el comportamiento de los actores.

El grado de desconfianza entre los diferentes actores respondía a que cada grupo defendía sus propios intereses. Un deficiente empoderamiento de todos los actores puede estar en el origen de la misma, mientras que el devenir histórico pasa por la democratización del conocimiento (Requena Mora y Rodríguez Victoriano, 2017: 11).

El liderazgo fue ejercido, fundamentalmente, por dos personas: un político (consejero de Sanidad) y un profesional/gestor (gerente de Área), lo que puede considerarse un error, pues al desaparecer estas personas el modelo entró en declive. La organización política y la gestora deberían haber asumido el liderazgo, explicando el modelo participativo (transparencia en la comunicación) y dedicando más recursos a la formación de los ciudadanos y de los profesionales, ya que estos son elementos muy importantes en la democracia.

Otros aspectos que habrán podido influir en el desarrollo del proyecto han sido los relacionados con la comunicación, tanto interna como externa. Problemas no tan difíciles de resolver si existe voluntad política para hacerlo.

Como conclusión final y recomendación, consideramos que el modelo participativo implantado en el área sanitaria de Puertollano es viable, siempre y cuando se aborden adecuadamente las debilidades y amenazas identificadas en esta investigación. Habría que recuperarlo aplicándolo a todo el territorio de Castilla-La Mancha, con el fin de avanzar en una democracia deliberativa, es decir, de alta intensidad democrática.

Revisado por José Morales

\section{Referencias bibliográficas}

AA.VV. (2008), "Indicadores de participación ciudadana y evaluación de procesos participativos”. Consultado el 09.10.2017, en http://www.redcimas.org/wordpress/ wp-content/uploads/2012/08/m_GobLaPaz_INDICADORES.pdf.

Ackerman, Bruce; Fishkin, James S. (2004), Deliberation Day. New Have, CT: Yale University Press.

AEVAL - Agencia Estatal de Evaluación de las Políticas Públicas y la Calidad de los Servicios (2010), "Fundamentos de evaluación de políticas públicas”. Madrid: Ministerio de Política Territorial y Administración Pública. 
Atanasova-Pieterse, Desislava; Kerekovska, Al (2013), "Barriers and Facilitators for Citizen Participation in Healthcare Decision Making at the Local Level in Bulgaria (Bulgaria, 2009)", European Journal of Public Health, 23(supl.1), 265-266.

Beste, Simon (2013), "Contemporary Trends of Deliberative Research: Synthesizing a New Study Agenda”, Journal of Public Deliberation, 9(2), 1-44.

Bispo, José Patricio; Gerschman, Silvia (2015), "Legitimidade da representação em instâncias de participação social: o caso do Conselho Estadual de Saúde da Bahia, Brasil", Cadernos de Saúde Pública, 31, 183-193.

Bleda García, José María; Aguilar Gil, Marta (2011), Participación ciudadana en salud. Madrid: Universitas.

Bleda García, José María; Aguilar Gil, Marta (2014), "Participação cidadã na saúde: órgãos de participação deliberativa na área de cuidados de saúde de Puertollano (Ciudad Real)", in Mauro Serapioni; Ana Raquel Matos (comps.), Saúde, participação e cidadania. Experiências do Sul da Europa. Coimbra: CES/Almedina, 133-154.

Bleda García, José María; Aguilar Gil, Marta (2016), "Participación ciudadana em el área de salud de Puertollano (Ciudad Real-Espanha): sueños e realidades”, O Público e o Privado, 27, 99-118.

Bohman, James (2009), "La democracia deliberativa y sus críticos". Consultado el 17.10.2017, en http://espacioagon.blogspot.com/2009/02/james-bohman-lademocracia-deliberativa.html.

Bustamante Ospina, Edilson (2013), "La comunicación interna en el Sistema Nacional de Salud en España”, Folios, 28, 139-160. Consultado el 16.10.2018, en http:// aprendeenlinea.udea.edu.co/revistas/index.php/folios/article/view/15107.

Cohen, Joshua (2001), "Democracia y libertad”, in Jon Elster (comp.), La democracia deliberativa. Barcelona: Gedisa, 236-287.

Donoso, Nora (2009), "Participación y ciudadanía activa en salud". Ponencia presentada en el Seminario Internacional "Participación en salud y gestión pública", Santiago de Chile, Chile.

Ginés Sánchez, Xavier; Requena Mora, Marina; Rodríguez Victoriano, José Manuel; Xambó Olmos, Rafael (2010), "Democratitzant la democràcia: la participació ciutadana des de baix". Ponencia presentada en el I Congreso Valenciano de Sociología, el 29 y 30 de enero, en la Universidad de Valencia.

Habermas, Jürgen (2005), "Tres modelos de democracia. Sobre el concepto de una política deliberativa”, Polis Revista Latinoamericana, 10. Consultado el 17.10.2017, en http://journals.openedition.org/polis/7473.

Hansen, Kasper M.; Rostbøll, Christian F. (2012), “Deliberative Democracy”, in Benjamin Isakhan; Stephen Stockwell (comps.), The Edinburgh Companion to the History of Democracy. Edinburg: Edinburgh University Press, 502-512.

Held, David (2008), Modelos de democracia. Madrid: Alianza Editorial. Ibáñez, Jesús (1997), A contracorriente. Madrid: Siglo XXI Editores. 
Jorba, Laia; Anduiza, Eva (2009), "Por qué y cómo evaluar la participación”, in Marc Parés i Franzi (coord.), Participación y calidad democrática. Evaluando las nuevas formas de democracia participativa. Barcelona: Ariel, 139-166.

Légaré, France; Thompson-Leduc, Philippe (2014), “Twelve Myths about Shared Decision Making”, Patient Education and Counseling, 96, 281-286.

López Giraldo, Luis Alirio (2008), "El rol de los actores decisores, corporativos y académicos en la formulación de políticas públicas de salud en Colombia”, in Gloria Molina Marín; Gustavo Alonso Cabrera Arana (comps.), Políticas públicas en salud: aproximación a un análisis. Colombia: Facultad Nacional de Salud Pública Héctor Abad Gómez, Universidad de Antioquia, 75-88.

Macpherson, Crawford Brough (1997), La democracia liberal y su época. Madrid: Alianza Editorial.

Manin, Bernard; Przeworski, Adam; Stokes, Susan C. (2006), "Eleições e representação", Lua Nova, 67, 105-138.

Matos, Ana Raquel; Serapioni, Mauro (2017), “The Challenge of Citizens' Participation in Health Systems in Southern Europe: A Literature Review”, Cadernos de Saúde Pública, 33(1).

Mosquera, Janeth; Gutierrez, Alejandra; Serra, Mauricio (2009), "La experiencia de participación ciudadana en el control social a la gestión en salud en Cali, Colombia”, Colombia Médica, 40(1), 95-102.

Ogando, Olga; Miranda, Belén (2001), "La evaluación de las políticas públicas: aspectos metodológicos y estudio de casos", VII Seminario "Evaluación y control de políticas públicas. Indicadores de gestión”. Gijón: Ayuntamiento de Gijón, 8-38. Consultado el 22.11.2017, en https://www.gijon.es/multimedia_objects/ download?object_id=88125\&object_type=document.

Parés i Franzi, Marc; Castellà, Carola (2009), "Una propuesta de criterios de calidad democrática para evaluar los procesos participativos”, in Marc Parés i Franzi (coord.), Participación y calidad democrática. Evaluando las nuevas formas de democracia participativa. Barcelona: Ariel, 241-266.

Pires, Valdemir; Pineda Nebot, Carmen (2011), "Presupuestos Participativos (PPs) e Instituciones Participativas (IPs) en Brasil: criterios (y marco) para la evaluación de experiencias y casos", REALA - Revista de Estudios de la Administración Local y Autonómica, 317, 261-280.

Requena Mora, Marina; Rodríguez Victoriano, José Manuel (2017), “Más allá de la democracia representativa: la democracia real y los movimientos sociales en el Estado español”, Revista Crítica de Ciências Sociais, 113, 3-28. DOI: 10.4000/ rccs.6642.

Richer, Madeleine (2005), "Participación y organización comunitaria en el sector salud: servicios sociales quebequense”, Revista de Ciencias Sociales, XI(2), 347-359. 
Robles, José Manuel (2008), "La democracia deliberativa y la democracia digital. El caso de QOT”, IDT - Revista de Internet, Derecho y Política, 7. Consultado el 16.10.2018, en http://www.redalyc.org/articulo.oa?id=78813265004.

Santana, Silvina; Lausen, Berthold; Bujnowska-Fedak, Maria; Chronaki, Catherine E.; Prokosch, Hans-Ulrich; Wynn, Rolf (2011), "Informed Citizen and Empowered Citizen in Health: Results from an European Survey", BMC Family Practice, 12:20. Consultado el 12.01.2018, en https://bmcfampract.biomedcentral.com/ articles/10.1186/1471-2296-12-20.

Santos, Boaventura de Sousa (2005), Democratizing Democracy: Beyond the Liberal Democratic Canon. London: Verso.

Segura, Andreu (2010), "La participación ciudadana, la sanidad y la salud", Gestión Clínica y Sanitaria, 12(2), 55-62.

Serapioni, Mauro; Duxbury, Nancy (2012), "Citizens Participation in the Italian Health-Care System: The Experience of the Mixed Advisory Committees", Health Expectations, 17, 488-499.

Serapioni, Mauro; Romaní, Oriol (2006), "Strengths and Challenges of citizens' participation in Local Health Councils: The Cases of Italy, England, and Brazil”, Cadernos de Saúde Pública, 22(11), 2411-2421.

Sierra Caballero, Francisco (1998), "Función y sentido de la entrevista cualitativa en investigación social”, in Jesús Galindo Cáceres (coord.), Técnicas de investigación en sociedad, cultura y comunicación. México: Pearson, 277-346.

van de Bovenkamp, Hester M.; Trappenburg, Margo J.; Grit, Kor J. (2010), "Patient Participation in Collective Healthcare Decision Making: The Dutch Model”, Health Expectations, 13(1), 73-85.

Villalbí, Joan R.; Tresserras, Ricard (2011), "Evaluación de políticas y planes de salud", Gaceta Sanitaria, 25(supl. 1), 17-24.

White, Robert A. (2004), “Is 'Empowerment' the Answer? Current Theory and Research on Development Communication”, Gazette: The International Journal for Communication Studies, 66(1), 7-24.

Recibido: 24.01.2018

Aceptación comunicada: 14.05 .2018

\section{Marta Aguilar Gil}

Departamento de Sociología, Universidad de Sevilla

C/ Pirotecnia, s/n. C.P. 41013 Sevilla, España

Contacto: maguilar6@us.es 
José María Bleda García

Facultad de Ciencias Jurídicas y Sociales, Universidad de Castilla-La Mancha

C/ Cobertizo San Pedro Mártir, s/n. CP. 45071 Toledo, España

Contacto: jose.bleda@uclm.es

The Model of Citizen Participation in Health Services in Puertollano (Spain): Beyond Political Will and Citizen Empowerment

A model for citizen participation in the area of health services was established in Puertollano (Castilla-La Mancha, Spain) and was carried out from 2008 to 2011. This was an innovative, prestigious and legally established model, based on the active and deliberative participation of the different actors involved in the health care system. In 2011 the conservative government that was formed after the regional elections halted all the actions taken under this model. As a result, we have considered it necessary to evaluate the model at the end of this period, carrying out a rigorous, systematic and scientific analysis of what took place during the years 2008-2015, examining the weaknesses in the application of the model and the possible threats it posed. For our analysis we have used a qualitative method based on in-depth interviews with actors involved in establishing the model.

Keywords: citizen participation; democracy; health systems; public policies; Spain.
Le modèle de participation citoyenne en matière de santé à Puertollano (Espagne): au-delà des décisions politiques et de l'autonomisation citoyenne

Le modèle de participation citoyenne en matière de santé mis en place dans la zone d'intervention sanitaire de Puertollano (Castille-La Manche, Espagne) eut lieu au cours des années 2008-2011. Il s'agit d'un modèle innovateur, prestigieux et consacré, reposant sur la participation active et délibérative de différents acteurs impliqués dans le système de santé. En 2011, le gouvernement conservateur issu des élections régionales a bloqué toutes les actions ayant trait au modèle en cause. Par conséquent, nous avons estimé qu'il convenait de procéder à une évaluation a posteriori à l'issue de la période de mise en œuvre, dans le but d'effectuer une analyse rigoureuse, systématique et scientifique de ce qui a eu lieu durant les années 2008-2015, en mettant en exergue les faiblesses et les menaces de l'application du modèle. À des fins de recherche, nous avons utilisé une méthodologie qualitative s'appuyant sur des entretiens approfondis d'acteurs engagés dans la mise en place du modèle.

Mots-clés: démocratie; Espagne; participation citoyenne; politiques publiques; systèmes de santé. 